\title{
ON THE APPLICATION OF PARTICLE SWARM OPTIMIZATION IN MINIMUM TIME LAUNCH VEHICLE TRAJECTORY OPTIMIZATION PROBLEM
}

\section{ADITYA CHOWDHURY \& VISHNU G NAIR}

Department of Aeronautical and Automobile Engineering, Manipal Institute of Technology, Manipal, Karnataka, India

\section{ABSTRACT}

The application of Particle Swarm Optimization (PSO), in solving minimum time trajectory optimization problem, studied in this paper. The ascent phase of a launch vehicle trajectory is considered, due to its highly nonlinear nature. It is one of the challenging problems in optimization scenario. Achieving the target in minimum time and error, satisfying all the constraints is the major objective. A highly nonlinear complex system, launch vehicle, is considered with the control parameter as its angle of attack. It is proved that, PSO approach outperforms other such type of optimization, procedures in trajectory optimization scenario, by providing better accuracy. The numerical results are evaluated, analyzed and presented in a MATLAB simulation environment.

KEYWORDS: Minimum Time Problem, Trajectory Optimization \& Particle Swarm Optimization (PSO)

Received: Aug 07, 2017; Accepted: Sep 27, 2017; Published: Oct 13, 2017; Paper Id.: IJMPERDOCT201736

\section{INTRODUCTION}

The launch vehicle trajectory is generally optimized, to meet the target by minimum fuel, minimum control or minimum time. The objective of optimization considered in this research, is to optimize the time taken by the launch vehicle to reach the right injection point, satisfying all the set constraints. This process provides an economic consideration, as well as performance enhancement. There is a vast amount of literature available, in the area of launch vehicle trajectory optimization.

Lukkana et al. [1], gives a detailed discussion on the minimum control approach, for the launch vehicle and in Mauro et. al. [2], a multi-stage launch vehicle trajectory is discussed. Many numerical procedures exist to solve the optimization problem. Gradient restoration algorithm [3] and shooting method [4], are the indirect methods, whereas direct collocation [5] and differential inclusion [6], are the few examples of direct methods. One of the standard procedures, to solve the nonlinear equations is a gradient or steepest-decent method. In Sirisha et al. [8] this method is applied, to a hyper-sonic launch vehicle. In Vathsal, et al. [9] min-max technique is used for a satellite launch system, to obtain an optimum pitch angle, so as to maximize the apogee velocity at a constraint altitude and perigee point values.

The evolutionary bio inspired techniques are very effective in solving such problems, due to their easy to handle nature. The implementation of such algorithms is comparatively less complicated [22]. The PSO algorithm proposed by Kennedy and Eberhart, in 1995 proved to be one of the best candidates, for solving stochastic problems. The algorithm works on the basis of best behavior, among various entities in a social system. The previous paper published by the authors, gives an insight on solving the same optimization problem, using 
non-evolutionary method such as steepest descent. In this paper, a comparison is also performed and it is clearly identified that, PSO algorithm outperforms steepest descent method, in solving minimum time problems that is considered in this paper.

The rest of the paper is organized as follows. An overview of the optimization problem formulation is discussed in section II. PSO and its application in the proposed system are given in section III. In section IV, simulation results and analysis are discussed, followed by conclusions inferred in section $\mathrm{V}$, along with some views and future scope.

\section{PROBLEM FORMULATION}

This section gives details about the mathematical modelling of the launch vehicle equation of motion. [21].

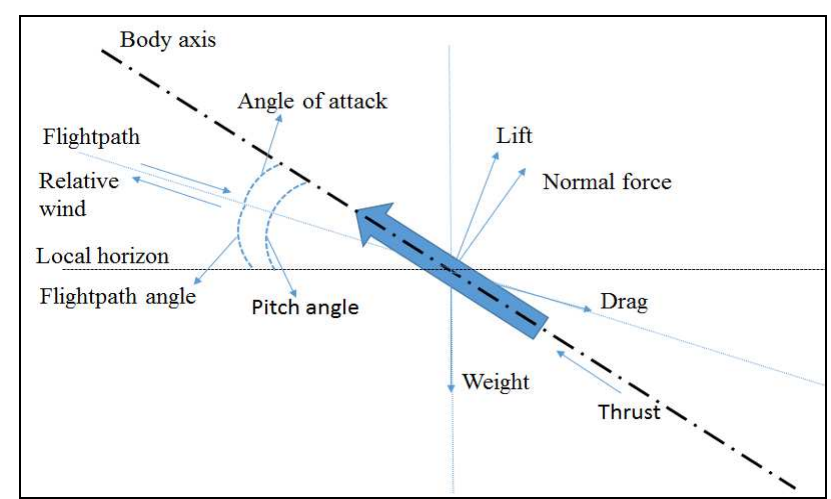

Figure.1: Force Components for an Air/Space Vehicle [21]

\section{Dynamics of the System}

As explained earlier, a highly nonlinear system, a launch vehicle is considered in this research. With reference to the available literature, a reference systems Advanced Launch vehicle System model (ALS), developed by NASA [19] is taken for the analysis, for the proposed methodology. Point mass equation of motion with spherical earth is given by,

$$
\begin{aligned}
& \dot{r}=V \sin \gamma \\
& \dot{V}=\frac{1}{m V}(T \cos \alpha-D-m g \sin \gamma) \\
& \dot{\gamma}=\frac{1}{m V}(T \cos \alpha+L)+\left(\frac{V}{r}-\frac{g}{V}\right) \cos \gamma
\end{aligned}
$$

Where, $\mathrm{m}, \mathrm{T}, \mathrm{D}$ and $\mathrm{L}$ refers to the mass of the launch vehicle, thrust, drag and lift, respectively, and $\mathrm{g}$ is the acceleration due to gravity.

The parameters $\mathrm{r}$ and $\mathrm{v}$, distance from centre of the earth and velocity, respectively and the flight path angle are the state variables, along with the angle of attack as the control parameter.

In this paper, minimum time optimization is considered. For that, the time dependent equations have to be converted into flight path angle dependent, so as to make flight path, a dependent variable.

$$
\dot{r}=F(\gamma) ; \dot{v}=G(\gamma) ; \gamma=I(\gamma)
$$




\section{Physical Constraints}

Constraints refer to functions that define the allowable limits of the respective variables. The constraints considered in this research are stated below.

- At the injection point, there must be a minimum error in state variables considered. Also, the accuracy of the achieved velocity, with respect to the desired one should be very high.

- Since, this is a minimum time optimization approach, the trajectory generated must be in such a way that, the vehicle reaches the injection point, in minimum possible time.

\section{Performance Measure}

The performance measure is selected, for evaluating the system performance and is denoted as $\mathbf{J}$ throughout the paper, which is also called as cost function. Here, the problem is a combination of minimum-time and minimum terminal error problems; the performance measure is given by [12]:

$$
J=\sum S_{X}\left\|x_{f}-x_{t_{f}}\right\|_{2}^{2}+\int_{t_{0}}^{t_{f}} d t
$$

Where, $s_{r} s_{v}$ and $s_{\gamma}$ are the weighing factors. They are properly selected for each constraint. To reformulate the performance measure, $\gamma$ is chosen as an independent variable. Therefore, the cost function can be substituted as,

$$
J=\sum S_{X}\left\|x_{f}-x_{\gamma_{f}}\right\|_{2}^{2}+\int_{\gamma_{0}}^{\gamma_{f}} \frac{d t}{d \gamma} d \gamma
$$

\section{PARTICLE SWARM OPTIMIZATION ALGORITHM FOR SOLVING MINIMUM TIME PROBLEM}

PSO is proposed by Kennedy and Eberhart in 1995, based on the behavior of social systems, such as fish schooling, bird flocking etc. Since, it is taking the best of all the entities in the system, it is better than other stochastic methods. It is one of the widely accepted and applied evolutionary optimization methodologies.

Consider that, $i$ represents the total number of particles and $\mathrm{D}$ is the degree of freedom. $i^{\text {th }}$ particle in $\mathrm{D}^{\text {th }}$ dimension is denoted as, $\mathrm{X} i=\left(x_{i 1}, x_{i 2}, \ldots . x_{i \mathrm{D}}\right)^{\mathrm{T}}$. The velocity of each particles are represented by, $\mathrm{V} i=\left(v_{i 1}, v_{i 2}, \ldots . v_{i \mathrm{D}}\right) \mathrm{T}$. Each particle has individual personal best positions and is denoted as, $\mathrm{P} i=\left(p_{i 1}, p_{i 2}, \ldots, p_{i \mathrm{D}}\right)^{\mathrm{T}}$. The best value obtained in each iteration is called the global best solution (' $\mathrm{g}$ ' in the equations).

Equation (7) is used to update the velocity and position of the particles in global, version PSO, in which the superscripts denote the iteration number [16].

$$
v_{i}^{t+1}(d)=\omega v_{i}^{t+1}(d)+c_{1} \operatorname{rand}\left(p_{i}^{t}(d)-x_{i}^{t}(d)+c_{2} \operatorname{rand}\left(p_{g}^{t}(d)-x_{i}^{t}(d)\right) x_{i}^{t+1}(d)=x_{i}^{t}(d)+v_{i}^{t+1}(d)\right.
$$

Where, $x_{i}^{t}(d)$ and $v_{i}^{t}(d)$ denotes current position and velocity of the $d^{\text {th }}$ dimension of the $i^{\text {th }}$ particle, and $r$ denotes the uniformly distributed random number $U \in[0,1] . c_{1}$ and $c_{2}$ are positive acceleration constants. $\omega$ is the inertia weight.

Due to the parameter dependent nature and the constant inertia weight, the classical PSO suffers from local 
optima locking, which may even result in particles going out of the boundary. To alleviate these shortcomings, Eberhart et.al [17] proposed a new version of PSO called Adaptive PSO (APSO). In this version, the inertia weight parameter is initially given a higher value and it goes on diminishing, with each iteration. In APSO, the adaptive inertial weight is updated, based on the following equation.

$$
\omega^{t+1}=\omega_{\max }-\left(\frac{\omega_{\max }-\omega_{\min }}{\max \text { iter }}\right) \text { iteration }
$$

Generally $\omega_{\max }$ and Error! Bookmark not defined. values are usually fixed as 0.9 and 0.4 .

\section{SIMULATION RESULTS AND DISCUSSIONS}

The minimum time problem is solved, by minimizing terminal error of a constant thrust engine launch vehicle trajectory. The control variable considered is the angle of attack. It is always kept under the allowable limits, so as not to increase beyond the critical limit. Table 1 gives target parameters considered [19].

Table 1: Desired Performance Parameters

\begin{tabular}{|c|c|c|}
\hline Parameters & $\begin{array}{c}\text { Initial } \\
\text { Conditions }\end{array}$ & $\begin{array}{c}\text { Final } \\
\text { Conditions }\end{array}$ \\
\hline$r$ & 6377353 & 6438553 \\
\hline$v$ & 65 & 2630 \\
\hline$\gamma$ & 89.5 & 0 \\
\hline$m$ & 1523400 & 152052 \\
\hline$t$ & 0 & 150 \\
\hline
\end{tabular}

The simulation is done in Matlab environment and corresponding results are given below. The best solutions obtained after doing 50 iterations are presented in figures 2 to 5. The parameters for the PSO are taken from Mengqi et al. [20]. Figure 2, represents the variation of control vector, with respect to time. Figure 3, shows the velocity attained by the launch vehicle, during each time instant of its flight. From the graph, it is achieving the target velocity in 147.6 seconds. Figure 4 and 5, represents the variation of flight path angle and the radial distance from centre of earth, respectively. From the results it is clear that, the injection criteria are met satisfactorily.

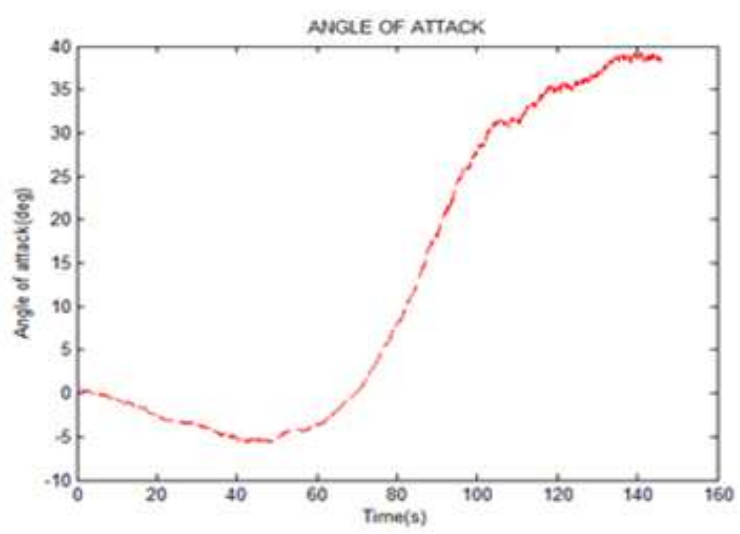

Figure 2: Variation of Control Variable 


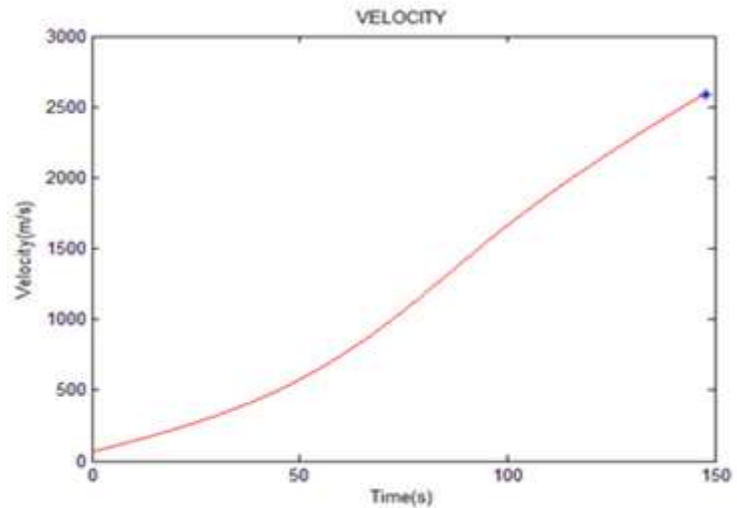

Figure 3: Velocity Profile of the Launch Vehicle

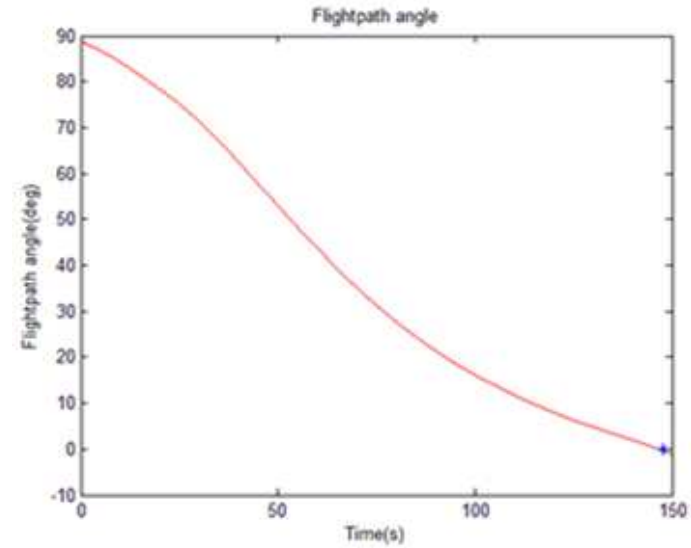

Figure 4: Variation of Flight Path Angle

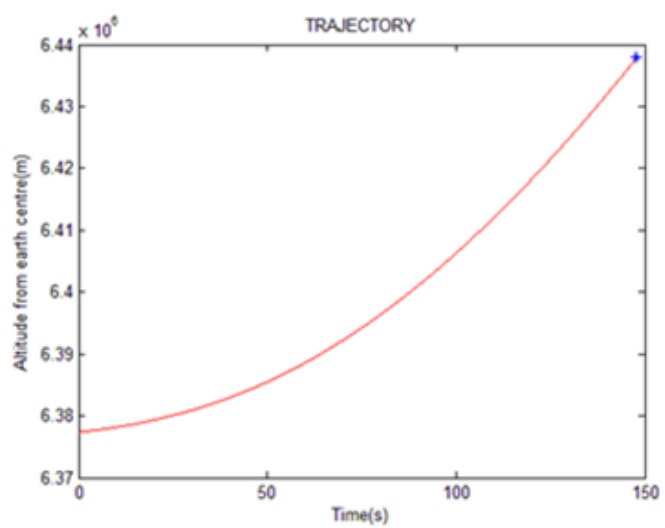

Figure 5: Radial Distance Variation

Table 2, shows the numerical analysis of the above results. To analyze the consistency and accuracy of PSO approach, the mean and standard deviation are also calculated for each variable. It is evident from the table that, the desired injection criteria, as per table 1 , are achieved satisfactorily. 
Table 2: Numerical Results

\begin{tabular}{|c|c|c|c|c|c|}
\hline Variables & $\Delta x_{f}$ & $\Delta x_{t_{f}}$ (best) & $\Delta x_{t_{f}}$ (mean) & $\Delta x_{t_{f}}$ (st.d) & Units \\
\hline$t$ & 5 & 147.6 & 148.8 & 0.94 & $s$ \\
\hline$r$ & 20 & 8.684 & 16.745 & 2.7485 & $m$ \\
\hline$v$ & 10 & 1.684 & 8.8539 & 4.8943 & $m / s$ \\
\hline$\gamma$ & - & 0 & 0 & 0 & $\mathrm{deg}$ \\
\hline
\end{tabular}

\section{CONCLUSIONS}

The application of Particle Swarm Optimization (PSO), in solving minimum time trajectory optimization problem is studied in this paper. The gravity turn trajectory is formulated for giving initial and final conditions. The target is achieved by minimum time and minimum error, at the injection point. Appropriate constraints are followed throughout the trajectory and necessary boundary conditions are applied. The PSO approach is implemented, for solving the complex nonlinear equations and the corresponding results, obtained through 50 iterations were validated. From the results, it is confirmed that, the injection parameters were met satisfactorily, as the future work hybrid approaches can be implemented and performance can be analysed.

\section{ACKNOWLEDGMENT}

The authors would like to acknowledge the support obtained from Manipal Institute of Technology, Manipal University for the successful completion of this research work.

\section{REFERENCES}

1. Lukkana Varaprasad, Radhakant Padhi “Ascent Phase Trajectory Optimization of a Generic Launch Vehicle” Xxxii National Systems Conference, IIT-Roorkee. NSC 2008

2. Mauro Pontani and Giampaolo Cecchetti "Ascent trajectories of multistage launch vehicles: numerical optimization with second order condition verification” Operations Research Article ID 498765, 2013

3. Miele and T. Wang “Multiple-subarc gradient-restoration algorithm, part 1: algorithm structure," Journal of Optimization Theory and Applications, vol. 116, no. 1, pp. 1-17, 2003.

4. K. R. Brown, E. F. Harrold, and G. W. Johnson "Rapid optimization of multiple-burn rocket flights," NASA Contractor Reports, 1969.

5. C. R. Hargraves and S. W. Paris “Direct trajectory optimization using nonlinear programming and col-location,” Journal of Guidance, Control, and Dynamics, vol. 10, no. 4, pp. 338-342, 1987.

6. H. Seywald. "Trajectory optimization based on differential inclusion," Journal of Guidance, Control, and Dynamics, vol. 17, no. 3, pp. 480-487, 1994

7. P. Chandra Sekhar, Unit Commitment of Thermal Generating Units Using Advanced Particle Swarm Optimization Techniques, International Journal of Electrical and Electronics Engineering Research (IJEEER), Volume 3, Issue 3, July August 2013, pp. 15-26

8. John T. Betts - Survey of Numerical Methods for Trajectory Optimization\| Journal Of Guidance, Control, And Dynamics Vol. 21, No. 2, AIAA 1998.

9. C. V. Sirisha and M. Manickavasagam "A Specific Energy Based Guidance Algorithm for the Hyper-sonic Launch Vehicle", 
International Conference on Avionics Systems Conference, RCI, Hyderabad, 2008

10. S. Vathsal, K.A.P. Menon and R. Swaminathan. "Minmax Approach to Trajectory Optimization of Multistage Launch Vehicles”, IEEE Transactions on Aerospace \& Electronic Systems, Volume AES-13, Issue 2, March 1977, pp 179-187

11. D. E. Krik." Optimal Control Theory: An Introduction", Prentice Hal, 1970

12. Joshi, Ashok, and B. Sudhir Kumar. "Effect of Ini-tial Flight Path Angle Error and Control Constraint on the Optimized Ascent Trajectory of a Typical Launch Vehicle", 19th AIAA International Space Planes and Hypersonic Systems and Technologies Conference, 2014.

13. Martin S. K. Leung and Anthony J. Calise. "Hybrid approach to near-optimal launch vehicle guidance", Journal of Guidance, Control, and Dynamics, Vol. 17, No. 5,pp. 881-888. doi: 10.2514/3.21285,1994

14. Kennedy J, Eberhart R, Shi Morgan Y. Swarm intel-ligence. San Francisco: Kaufmann Publishers; 2001.

15. Trelea IC. The particle swarm optimization algo-rithm: convergence analysis and parameter selec-tion. Inform Process Lett; 85(6):317-25., 2003

16. Zhong W, Li S, Qian F. theta-PSO: a new strategy of particle swarm optimization. J Zhejiang Univ Sci A; 9(6): 786-90,2008.

17. M. Clerc and J. Kennedy, "The particle swarm-explosion, stability, and convergence in a multi-dimensional complex space", IEEE Trans. Evol Comput, vol. 6, no. 1,pp. 58-73,2002

18. R. C. Eberhart and Y. Shi, "Tracking and optimizing dynamic systems with particle swarms," in Proc. Congr. Evol. Comput.,, pp. 94-100,2001

19. Safari, H. Shayeghi, "Iteration particle swarm optimization procedure for economic load dispatch with generator constraints", Exp Syst Appl, vol. 38, (2011), pp. 6043-6048.

20. Ping Lu. "Nonlinear trajectory tracking guidance with application to a launch vehicle", Journal of Guidance, Control, and Dynamics, Vol. 19, No. 1 (1996), pp. 99-106. doi: 10.2514/3.21585.

21. Mengqi Hu, Teresa Wu, and Jeffery D. Weir “Adaptive Particle Swarm Optimization With Multi-ple Adaptive Methods" IEEE Transactions On Evolutionary Computation, Vol. 17, No. 5, October 2013

22. Diksha Diva Singh, Vishnu G. Nair and Dileep M. V, "Minimum Time Ascent Phase Trajectory Optimization using Steepest Descent Method", International Journal of Control Theory and Applications, Vol.9 (39), 2016, pp. 71-76.

23. Fernando Alonso Zotes; Matilde Santos Peñas, "Delta-V genetic optimization of a trajectory from Earth to Saturn with fly-by in Mars" IEEE Congress on Evolutionary Computation 2010 Pages: 6, DOI:10.1109/CEC.2010.55861 
